\title{
NEW DIMENTIONS OF SAFETY AND SECURITY OF SPECIAL OLYMPICS ATHLETES DURING EVENTS
}

\author{
Stefka Djobova, Ivelina Kirilova \\ National Sports Academy "Vassil Levski” (Bulgaria)
}

\begin{abstract}
There is no doubt that the COVID-19 pandemic has significantly affected the sport sector worldwide as well as sports calendar. Sports events have been cancelled or rescheduled like Summer Olympic and Paralympic games. The same had happened with the Special Olympics (SO) events. The pandemic constitutes a global challenge to the sport movements in terms of security and safety for all participants. Much more complicated it appears to secure the athletes with intellectual disabilities. Pandemic research has found that people with intellectual disabilities are almost six times more likely to die from COVID-19 than the general population. The SO programs organizers will face unknown so far issues in terms of security and safety.

Aim: The aim of the study is to explore the influence of new safety and security events protocols of Special Olympics on the mission and particularly on the athletes.

Methods: We applied documentary analysis including theoretical-logical analysis, systematic approach (structural-functional, structural-component and structural-integral aspect), comparative analysis, video analysis, field notes and focus group composed by participants in pervious SO World games (delegation leaders, coaches and parents).

Results: The results of our research shows that most of the national SO programs are reporting that the pandemic have a devastating impact on the athletes and the movement in general. The new live reality is affecting not only the daily routines of athletes with intellectual disabilities but also their social interaction in terms of communication and emotional functioning.

Conclusion: Having in mind that safety is first priority and applying all protocols, $\mathrm{SO}$ is attempting to preserve the mission, to ensure joy of sport and physical activity. In the new reality close contact and friendships are approached in a way to preserve them and to keep them as essential part of the events.

Keywords: COVID 19; safety; security; Special Olympics athletes; intellectual disabilities; sport
\end{abstract}

\section{Introduction}

We are facing a global health crisis that we have not seen in the last 70 decades in the human history. World Health Organization has characterized the coronavirus 
disease, called COVID-19 as a pandemic. However, the situation is much more complicated - it is a human, economic and social crisis. Every aspect of people's lives is influenced. This virus is attacking not only humans' health but also the societies at their core (Guterres 2020). "Unlike any other virus or disease of this generation, COVID-19 does not adhere to national borders nor does it change its approach on economic grounds. Quite the contrary. COVID-19 does not discriminate, and it does not apologize" - strong words of Special Olympics Europe Eurasia President and Managing Director David Evangelista (Evangelista n.d.). Nevertheless, that the virus's outbreak affects all segments of the population, it is particularly detrimental to members of those social groups in the most vulnerable situations (UN 2020). Traditionally persons with disabilities are at higher risk of margination and social isolation and at highest risk are persons with intellectual disabilities (ID). People with ID are less likely to access health care, education, sport activities, employment and to participate in the community. Even under ordinary circumstance it is more likely to live in poverty, experience higher rates of violence, neglect and abuse, and are among the most marginalized in any crisis-affected community. COVID-19 has further compounded this situation, disproportionately influencing persons with disabilities both directly and indirectly (UN 2020a).

According to the research of Courtenay and Perera, people with ID are especially vulnerable to the physical, mental and social effects of the pandemic (Courtenay \& Perera 2020). Because of their specificity not only in physical health but in their habits, people with intellectual and developmental disabilities (IDD), such as Down Syndrome, ASD, etc. are at higher risk of contracting COVID-19 and also of experience serious illness associated with it (Grier et al. 2020). They may suffer from hereditary cardiac conditions, inborn errors of metabolisms and respiratory infections that are defined as the leading cause of death in people with ID, especially among people with Down syndrome (Baburamani et al. 2019, O'Leary et al. 2018, Sanyer 2006). According to CDC (2020) people how have complex medical problems as neurologic, genetic, metabolic conditions, or who have congenital heart disease are at higher risk for severe illness from COVID-19 than other population. In addition, many researches show that the level of obesity is higher among people with ID. This state is related major to their sedentary lifestyle, fragmental or missing physical activity and improper diet (Lloyd 2016, Phillips \& Holland 2011).

More than a year has passed since the beginning of this crisis, and recent studies examined how the pandemic has affected specific social groups as people with ID. A cross-sectional study conducted by Gleason et al. (2021) of 64,858,460 patients across 547 health care organizations, discloses that having an ID is the strongest independent risk factor for presenting with a COVID-19 diagnosis and the strongest independent risk factor other than age for COVID-19 mortality. The results also revealed that those with ID are 2.5 times more likely to contract COVID-19, were 
about 2.7 times more likely to be admitted to the hospital and 5.9 times more likely to die from the infection than the general population. Also, the authors discussed that beyond the direct risk of Covid-19, the pandemic has had negative effects on the ability of individuals with ID to receive the health care and daily support that they typically receive. (Gleason et al. 2021). Same conclusions reported many other studies (Landes et al. 2020, Makary 2020). According to Inclusion International network survey, $54 \%$ of respondents answered that government's response to COVID-19 was not inclusive for people with ID and their families. In addition, their opinion is that the pandemic has resulted in increased deaths of people in institutions and other segregated settings, isolation and decreased access to: supports, information and social protection measures.

From the distance of time, we can conclude that stakeholders and leading organizations rapidly realized the need of protection of all defined as vulnerable groups of society like those with intellectual disabilities. They quickly identified the most probable affected areas in the daily life of persons with ID and their families. As a result, they appeared with recommendations and guidelines first for public health authorities around the world about acting to contain the COVID-19 outbreak. After they addressed their policy to other affected areas as an education, labor market, human rights and economics as whole and sport sector. Of course, the realization of these recommendations differs around the world depend on many factors as economic status of the country, condition of public health system, the cultural and native specificities. Sport organizations also appear with their own recommendations. A global movement Special Olympics is launched more than sixty years ago, with a core aim to "break down barriers and end discrimination against people with intellectual disabilities" (Special Olympics n.d.). Nowadays SO is the largest sport organization for persons with ID. SO also appear with recommendations and take a policy regard protection lives, but not only of athletes as well as their families, coaches, volunteers and caregivers around the world. According to SO, form the beginning of pandemic they are "doing everything possible to prevent the spread of COVID-19 and provide resources and programming to support prevention and promote the health, safety, well-being, and engagement of the Special Olympics community as in-person events are postponed and while physical distancing is required" (Special Olympics n.d.a). They develop a variety of resources to help all involved with the movement as well as new safety and security events protocols for trainings and competitive events.

The aim of the study is to explore the influence of new safety and security events protocols of Special Olympics on the mission and particularly on the athletes.

\section{Methods}

We applied documentary analysis including theoretical-logical analysis, systematic approach (structural-functional, structural-component and structural- 
integral aspect), comparative analysis, video analysis, field notes and focus group composed by participants in pervious SO World games (delegation leaders, coaches and parents).

\section{Results}

At the first phase of our study we analyzed the key points of the mission of SO and we developed graphical model to facilitate our further research. (Figure 1)

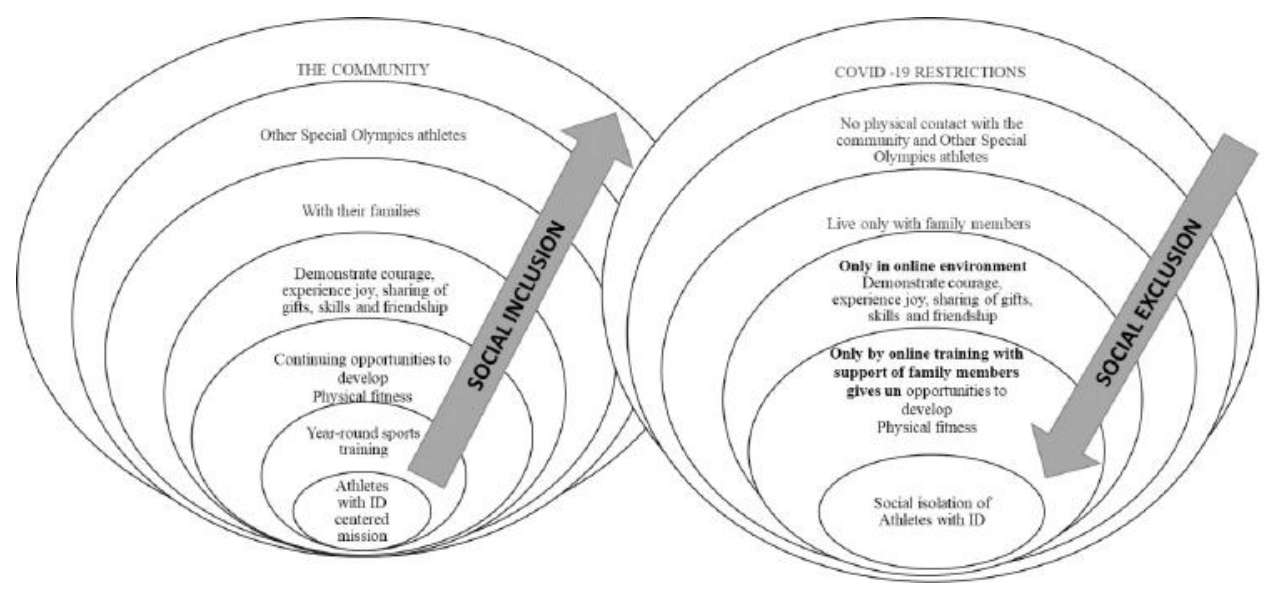

Figure 1. SO mission and COVID-19 impact model

The ultimate goal of SO is to change the worlds perception about persons with intellectual disabilities. No matter what the level of their personal development is, it is important to be demonstrated to the wider auditory. Every athlete is working hard continuously to achieve its personal goals in physical fitness and sports specific skills development. These results are accomplished under the appropriate supervision of qualified sport experts - coaches, instructors and many other volunteers who believe that every person matters. All athlete achievements are demonstrated during the sport events. These events are like no other because athletes are overcoming tremendous personal and social barriers in order to demonstrate courage and sporting excellence. In this adventure, they are not alone. The events of Special Olympics are offering platform for sharing friendships, skills, gifts, but more importantly to experience joy and happiness in environment where no one is left alone. This positive experience is shared with families, friends and coaches. It is also shared with other athletes from different nations. All these positive emotions are bringing the real sense of community where everyone is accepted. All milestones of the mission are complementing the dynamic process of social inclusion. In the real sense of sharing, everyone 
is a part of a big constant hug and high fives are instant part of every moment of appreciating. These amazing results have been accomplished gradually and year by year during last five decades. (Figure 2)

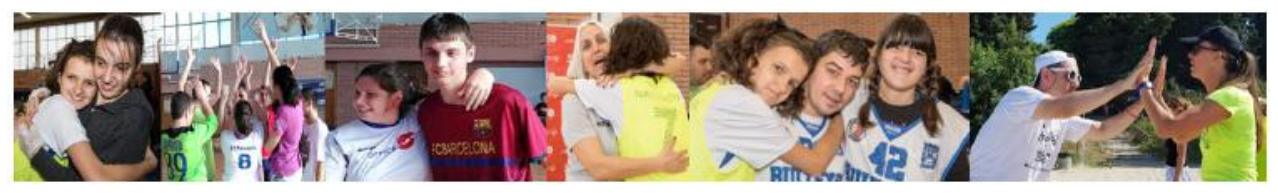

Figure 2. Sharing a friendship in Special Olympics

In 2020 the mankind was hit by the COVID-19 pandemic wave and the most vulnerable groups were the most badly affected. Immediate restriction measures were applied all over the world and for a short period the perspective looked really shocking. Our contacts from the focus group are reporting that those who were in the focus of inclusion were locked down and at the very beginning excluded also from excluded. The structured routine of the SO athletes was broken, no trainings were offered, no contacts with the friends. The social circle narrowed just to the family but still they had the support and the emotional affection from the parents. Critical appears the situation in the social institutions where due to the environment restrictions were more severe. The results of the document analysis are demonstrating that most of the national SO programs are reporting that the pandemic have a devastating impact on the athletes and the movement in general. According to the results from the focus group, there is a new live reality and this reality is affecting not only the daily routines of athletes with intellectual disabilities but also their social interaction in terms of communication and emotional functioning. In order to address the new reality issues SO develop rapidly own protocols and recommendations. SO approaches not only athletes but Unified partners, coaches, healthcare professionals, volunteers, family members, caregivers, staff or others in attendance of an activity - the surrounding society as whole. Documentary analysis reveal that the development of SO protocols is based on the expertise, knowledge and expertise of reliable organizations as World Health Organization (WHO) and US Center for Disease Control (CDC) as well numerous governments, sports governing bodies, and other organizations. At FAQ published on the SO website was reported that input was sought from Program CEOs, staff and athletes, legal, and risk management, human resources, programmatic leads (sport, health, youth/schools, leadership), Global Medical Advisory Committee, and Regional Presidents (Special Olympics 2021). In addition, they had review by World Athletics, International Paralympic Committee, CDC, US Olympics and Paralympic Committee and others. This is a hedge step in approaching 
the complexity of issues that are related to the specificity of people with ID behavior, sports activities and the differences in national COVID-19 level of restrictions. (Figure 3)

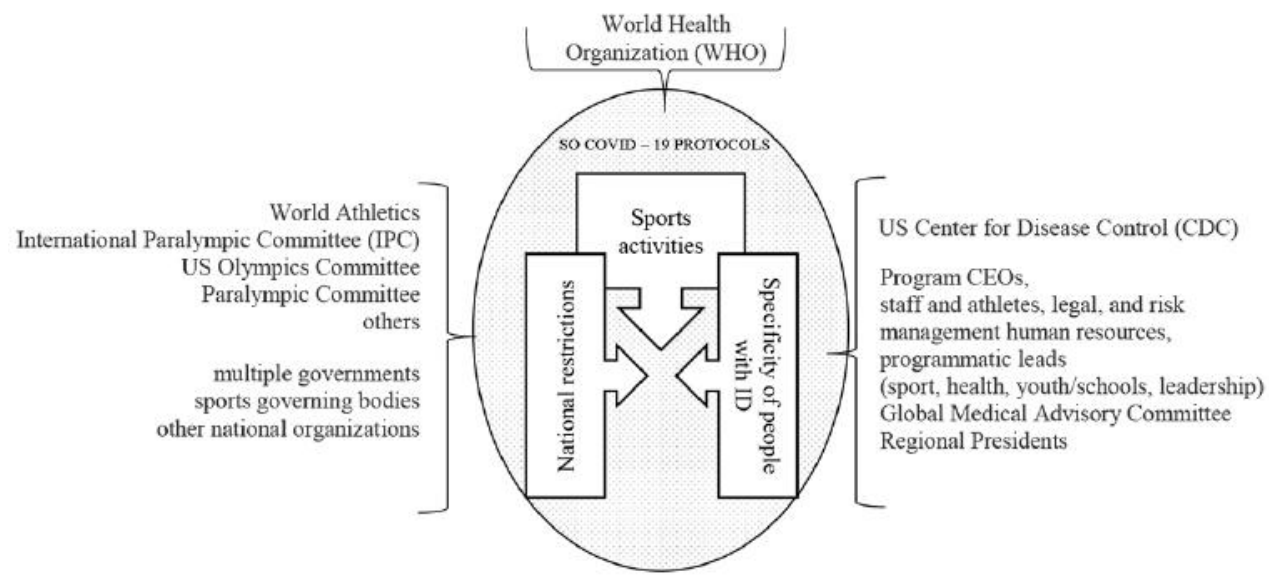

Figure 3. Special Olympics COVID-19 protocols

After a first and second wave of pandemic and finding hope in new invented vaccine slowly but sustainably the whole world tries to recover to the normal lifestyle. Special Olympics also develop Return to activities protocol (RAP) which include guidelines founded on the latest medical information available and guidance from organizations such as the World Health Organization (WHO) and nation-specific health agencies such as the Centers for Disease Control and Prevention (CDC) in the U.S. There are five guiding principals in RAP. The leading one is: "The health and safety of all members of the Special Olympics movement is paramount." Also, the guidance takes a phased approach. There are three phases to return to activities and each one is dependent on local transmission rates as well as testing/monitoring/contact tracing/health system capacity. Phase 0 - or stay at home - No events or activities of any sort to be held in person. Individual sport training sessions in own home using own equipment are allowed. The coaching occurs virtually. SO have developed and provide Fitness and Health programming offered virtually as School of Strength, Fit 5, Stay fit at home, Live fitness workout.

Also, a lot of educational resources and online live meetings, story-sharing page for and from SO athletes with lots of videos with positive messages. Other phases are providing rules depend on the local situation how safety to return to in person sport activities. 
In order to protect the integrity of the movement and to sustain the mission in relation to the subject of COVID-19, Special Olympics developed a set of statements that should be provided without additional comment or interpretation. SO also recommended to the staff to refrain from engaging in speculation or expounding about any issues of guidance. As socially responsible organization, Special Olympics is committed to doing everything possible to prevent COVID-19 spread. Athletes were provided with information on how to protect themselves from exposure to COVID-19. Videos and different at-home resources, including fitness videos to stay healthy and fit and emotional well-being graphics and webinars to reduce stress and enable stronger social connectedness were released. Athletes were properly addressed and equipped with tools for self-care. SO addressed also the frontline healthcare workers on how to treat people with ID.

\section{Discussion}

The ultimate goal of Special Olympics is to help persons with intellectual disabilities participate as productive and respected members of society at large, by offering them a fair opportunity to develop and demonstrate their skills and talents through sports training and competition, and by increasing the public's awareness of their capabilities and needs (Special Olympics n.d.b). At the same time people with ID are one of the most vulnerable populations to potential exposure to COVID-19. Suspension of training and activities affected some athletes much more than the epidemiological situation. Inclusion process went through different stages form slightly delayed to deeply frozen. To maintain the sense of belonging SO addressed the situation at different levels with major concern about the athletes and their wellbeing. Athletes define success mainly as confidence and assertiveness. Mobilization and belief in one's abilities is dominant (Iancheva, Kuleva 2017). Abilities development were addressed through variety of sets for distance or home-based training. Parents were motivated to become part of the training process. SO produced videos and used social media to share affection and personal success stories. Person-centered ideology is still remaining as a focal point at all levels. More than ever human rights as right to health care is brought to the front line. Physical activity is an important element and criterion for a healthy lifestyle (Borukova 2019). Numerous materials related to health literacy were produced and all channels were used to reach in the most effective way to the athletes. Unique symbiosis was observed among the nongovernmental organizations. A lot of protocols and guidelines were produced but few managed to address in meaningful way and reach the target group. We confirmed our previous findings that the sustainable programs, who have reached level of independent existence even in crisis are contributing to continuous self-development. (Djobova et al. 2020). As many were concerned about the exclusion, persons with intellectual disabilities 
included in SO programs were not forgotten. Once again it was proven that sport has the universal power to unite people, to overcome political, cultural and social barriers and to develop human personality.

\section{Conclusion}

Having in mind that safety is first priority we reviled that SO through the application of all the newly developed knowledge and experience-based protocols is attempting to preserve the mission, to ensure joy of sport and physical activity. In the new reality close contact and friendships are approached in a way to preserve them and to keep them as essential part of the events.

\section{REFERENCES}

Baburamani, A., Patkee, P., Arichi, T. \& Rutherford, M., 2019. New approaches to studying early brain development in Down syndrome. Developmental medicine \& child neurology. [viewed 14 May 2021]. Available from: https://onlinelibrary.wiley.com/doi/pdf/10.1111/ dmen. 14260

Borukova, M., 2019. Factor structure and major factors of physical ability of 13-14-year-old pupils. Journal of Applied Sports Sciences (1), 77 - 84. [viewed 21 May 2021]. Available from: doi.org/10.37393/jass.2019.01.7

CDC. 2020. People at Higher Risk for Severe Illness. [viewed 16 May 2021]. Available from: https://www.cdc.gov/coronavirus/2019-ncov/faq. html\#People-at-Higher-Risk-for-Severe-Illness

Courtenay, K. \& Perera, B., 2020. COVID-19 and people with intellectual disability: impacts of a pandemic. Irish Journal of Psychological Medicine, 37(3) Special Issue: Covid-19 perspectives, 231 - 236 [viewed 13 May 2021]. Available from: doi.org/10.1017/ipm.2020.45

Djobova, S., Borukova, M. \& Kirilova, I., 2020. Building social capital through inclusive basketball, Trakia Journal of Science, 18(1), 891 - 896. [viewed 21 May 2021]. Available from: doi.org/10.15547// tjs.2020.s.01.146

Evangelista D. S. n.d. COVID-19 Teaches Us about the Need for a Unified Approach to Health. [viewed 13 May 2021]. Available from: https:// www.specialolympics.org/stories/news/covid-19-teaches-us-about-theneed-for-a-unified-approach-to-health

Gleason, J., MD., Wendy Ross, MD., Fossi, A., Blonsky,H, Jane T. \& Mary, S., 2021. The Devastating Impact of Covid-19 on Individuals with Intellectual Disabilities in the United States. [viewed 17 May 2021]. Available from: https://catalyst.nejm.org/doi/full/10.1056/CAT.21.0051

Grier E., Lunsky Y. William F., Sullivan \& Casson I. 2020. Health care of adults with intellectual and developmental disabilities in a time of 
COVID-19. [viewed 14 May 2021]. Available from: https://www.cfp.ca/ news/cfpnews/2020/04/09/04-09-02.full.pdf

Guterres, A., 2020. The recovery from the COVID-19 crisis must lead to a different economy. [viewed 12 May 2021]. Available from: https:// webcache.googleusercontent.com/search?q=cache:uuPWvsR2XxEJ:htt ps://www.un.org/en/coronavirus/un-secretary-general+\&cd=2\&hl=bg\& $\mathrm{ct}=\mathrm{clnk} \& \mathrm{gl}=\mathrm{bg}$

Iancheva T. \& Kuleva M., 2017. Cope with success in sport. Journal of Applied Sports. (1), 46 - 57. [viewed 20 May 2021]. Available from: doi. org/10.37393/jass.2017.01.5

Landes, SD., Turk, MA. \& Wong A.W.W.A., 2021. COVID-19 outcomes among people with intellectual and developmental disability in California: The importance of type of residence and skilled nursing care needs. Disabil Health J. 14(2):101051. [viewed 23 May 2021]. Available from: doi.org/10.1016/j.dhjo.2020.101051

Lloyd, M., 2016. Physical Activity of Individuals with Intellectual Disabilities: Challenges and Future Directions. Current Developmental Disorders Reports. (3), 91 - 93. [viewed 16 May 2021]. Available from: https://doi.org/10.1007/s40474-016-0079-5

Makary, M., 2020. Risk Factors for COVID-19 Mortality among Privately Insured Patients. . [viewed 23 May 2021]. In: Risk Factors for COVID-19 Mortality among Privately Insured Patients - A Claims Data Analysis A FAIR Health White Paper.pdf

O'Leary, L., Cooper, SA., Hughes-McCormack, L., 2018. Early death and causes of death of people with intellectual disabilities: a systematic review. Journal of Applied Research in Intellectual Disabilities. 31(3), 325 - 342 [viewed 14 May 2021]. Available from: doi.org/ 10.1111/jar.12417

Phillips, AC. \& Holland, AJ., 2011. Assessment of Objectively Measured Physical Activity Levels in Individuals with Intellectual Disabilities with and without Down's Syndrome. PLoS ONE 6(12): e28618. [viewed 16 May 2021]. Available from:doi.org/10.1371/journal.pone.0028618

Sanyer, O.N. (2006). Down syndrome and sport participation. Current Sports Medicine Reports. (5), 315 - 318.

Special Olympics. 2021. Frequently Asked Questions: Special Olympics Return to Activities - COVID-19 [viewed 22 May 2021]. Available from: Frequently Asked Questions: Special Olympics Return to Activities COVID-19

Special Olympics. n.d. [viewed 25 May 2021]. Available from: https:// www.jointherevolution.org/

Special Olympics. n.d. a. [viewed 23 May 2021]. Available from: https:// www.specialolympics.org/our-work/covid19 
Special Olympics. n.d. b. Mission, Goal and Founding Principles of Special Olympics Article 1. [viewed 23 May 2021]. Available from: https:// resources.specialolympics.org/governance/special-olympics-generalrules/article-1 ?locale $=$ en

UN. 2020. Everyone Included: Social Impact of COVID-19. [viewed 13 May 2021]. Available from: https://www.un.org/development/desa/dspd/ UN. 2020a. 2020. Policy Brief: A Disability-Inclusive Response to COVID-19 [viewed 13 May 2021]. Available from: https://unsdg.un.org/ resources/policy-brief-disability-inclusive-response-covid-19

https://orcid.org/0000-0003-2792-0934

Ivelina Kirilova

https://orcid.org/0000-0003-2041-1213

Adapted Physical Activity and Sport Sector

Department of Water Sports

National Sports Academy "Vassil Levski"

E-mail: stefka.djobova@abv.bg 